\title{
La destruction séléctive des lieux par les « hooligans » de Belgrade
}

\section{Amaël Cattaruzza}

\section{OpenEdition}

\section{Journals}

\section{Édition électronique}

URL : http://journals.openedition.org/echogeo/2164

DOI : 10.4000/echogeo.2164

ISSN : 1963-1197

\section{Éditeur}

Pôle de recherche pour l'organisation et la diffusion de l'information géographique (CNRS UMR 8586)

\section{Référence électronique}

Amaël Cattaruzza, «La destruction séléctive des lieux par les « hooligans » de Belgrade », EchoGéo [En ligne], Sur le Vif, mis en ligne le 27 février 2008, consulté le 01 mai 2019. URL : http:// journals.openedition.org/echogeo/2164 ; DOI : 10.4000/echogeo.2164

Ce document a été généré automatiquement le 1 mai 2019.

\section{(c) (i) (9)}

EchoGéo est mis à disposition selon les termes de la licence Creative Commons Attribution - Pas d'Utilisation Commerciale - Pas de Modification 4.0 International 


\title{
La destruction séléctive des lieux par les " hooligans » de Belgrade
}

\author{
Amaël Cattaruzza
}

1 Le 17 février 2008 vers 16 h00, l'Assemblée nationale du Kosovo proclamait officiellement l'indépendance de leur République. Cette déclaration était attendue. La réaction de Belgrade l'était aussi. Le journal Politika ${ }^{1}$ dans son édition du week-end, ouvrait son numéro par un long texte d'analyse dont les premières phrases synthétisent le contenu : "Quel que soit ce qui se passe à Pristina ce soir, cela ne sera pas la fin d'une page de notre histoire, mais bien son commencement. Le début d'un combat, d'un marathon dans lequel ce qui est important n'est pas de partir le plus vite, mais d'arriver au but. L'indépendance que prévoit de proclamer l'Assemblée du Kosovo n'est pas la première menace de la sorte concernant ce territoire, et à chaque fois, chacune de ses tentatives ont fini tôt ou tard par le retour du Kosovo et Metohija dans son Etat-mère." (Vladimir Radomirovic, Politika, 17/02/2008, traduit par l'auteur).

Cette journée de dimanche, je l'ai passée, comme la plupart des Serbes, à suivre heure par heure, minute par minute, les évènements au Kosovo et en Serbie. Les principales chaînes de télévision et stations de radio avaient mobilisé leur rédaction toute la journée pour un programme spécial d'information, entrecoupé d'analyses de politologues et d'hommes politiques, et de chants folkloriques serbes de la région du Kosovo et Metohija. J'ai vécu en direct la déclaration du président démocrate Boris Tadic déclarant que la Serbie ne reconnaîtrait jamais le Kosovo, appelant la population à réagir pacifiquement aux évènements actuels, et demandant aux forces internationales présentes sur place d'assurer la sécurité des Serbes et de toutes les communautés non-albanaises du Kosovo. Quelques minutes plus tard, c'était au tour du premier ministre nationaliste Vojislav Kostunica d'intervenir. Son discours était moins feutré. Tout en appelant au calme face à la proclamation de cet "Etat fantoche" [lazna drzava], il désignait les responsables : "Les Etats-Unis ont décidé et l'Europe a courbé la tête". Plus loin, il précisait : "nous voyons aujourd'hui les buts réels des bombardements de la fin des années 90" (photo1). Le cadre était posé. 
Photo 1 - Ancien ministère de la défense bombardé en 1999 par l'OTAN en plein centre de Belgrade

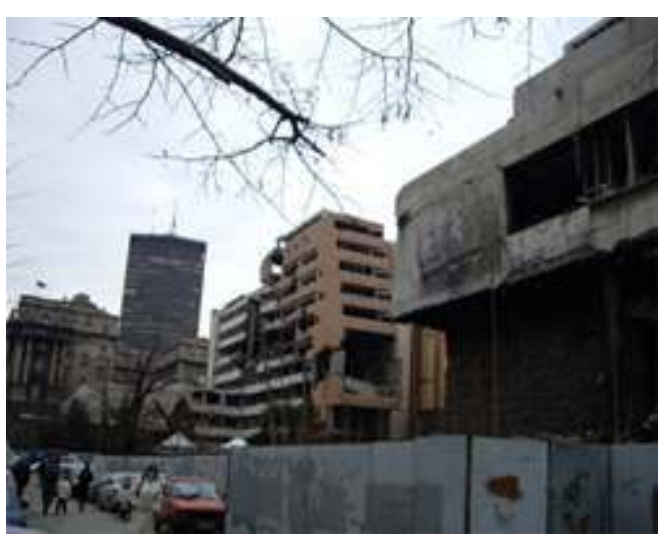

Dès 18 h00 à Belgrade, une bande de cinquante, cent, puis deux cents hooligans ${ }^{2}$, la plupart mineurs ou tout juste majeurs, se réunissent devant l'Ambassade des Etats-Unis. Après avoir scandé pendant une petite heure "Le Kosovo est la Serbie", ils commencent à lapider la façade, et alors s'engage une confrontation avec les forces de l'ordre, faisant une cinquantaine de blessés des deux côtés. Cela dura jusqu'aux environs de minuit, en plein coeur de Belgrade. Le lundi matin, l'inventaire des dégâts dans la ville était significatif. Parmi les autres lieux pris pour cibles par les hooligans, ceux qui évoquent la puissance américaine ou les pays européens qui se préparent à reconnaître l'indépendance, ou qui ont un quelconque lien avec les Albanais : le Mac Donald de Terazije (photo), qui a été incendié, le magasin Benetton (photo) et une boulangerie dont le propriétaire aurait de la famille albanaise au Kosovo, tous les trois au centre de Belgrade. Dans un contexte de crise, nous voyons que les lieux prennent un sens particulier et sont porteurs d'un message symbolique. Ces bandes de jeunes qui se regroupent et agissent de manière (en apparence) improvisée sont porteuses d'un message clair visible dès le dimanche soir dans le choix de leur cible. Ici, la géographie des lieux attaqués a du sens.

Photos 2 et 3 - Restaurant Mac Donald et boutique Benetton attaqués le dimanche 17 février au soir

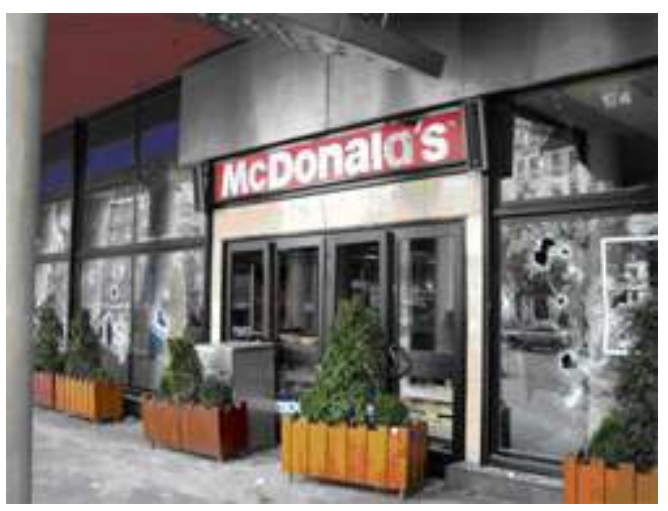




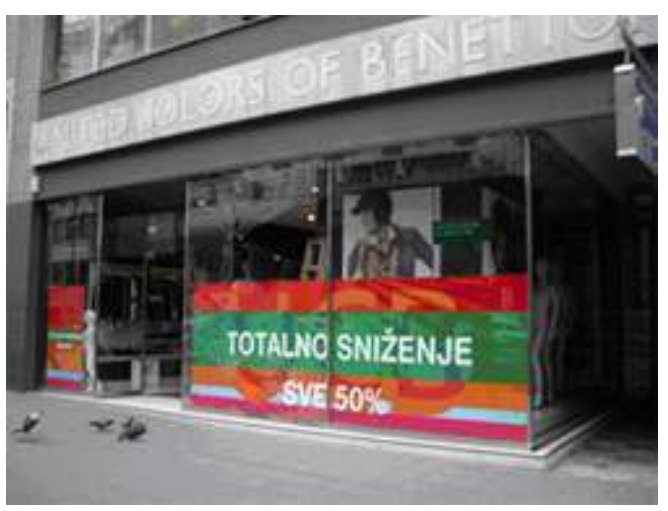

4 Cette destruction sélective a continué et s'est intensifiée dans la journée de lundi. Cette fois-ci, les hooligans ont profité des manifestations pacifiques organisées dans le pays pour se fondre aux manifestants et intervenir sur des sites précis. Un deuxième Mac Donald's au centre de Belgrade a été attaqué dans l'après-midi. Depuis, tous les restaurants de la firme sont fermés et font quelquefois l'objet d'une protection policière. Par ailleurs, plusieurs mosquées ont été endommagées à Belgrade et à Nis, ainsi que l'Ambassade de Turquie. Enfin, un drapeau slovène a été brûlé devant l'Ambassade de Slovénie et plusieurs drapeaux américains et albanais ont subi le même sort dans le pays. La Slovénie est ici prise à parti du fait de son rôle, en tant que présidente de l'Union Européenne, dans l'organisation de la mission de supervision européenne au Kosovo, EULEX (photo 4). De même que l'ambassade, les centres commerciaux slovènes Mercator ont été visés et ont fait l'objet de blocages par des manifestants.

Photo 4 - Affiches de protestation contre la mission européenne, "mission=occupation"

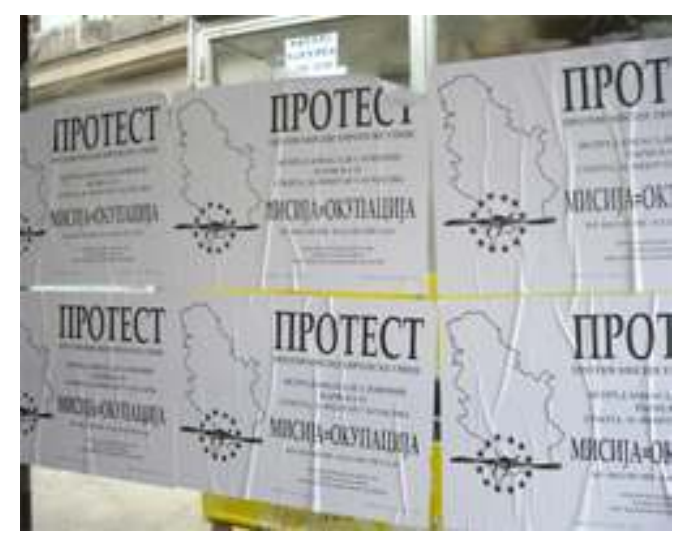

Enfin, le climax des destructions est atteint le jeudi 22 février au soir. Les principaux partis serbes en Serbie, au Kosovo, au Monténégro et en Republika Srpska (BosnieHerzégovine), dans une atmosphère d'Union nationale serbe, avaient appelé à une manifestation pacifique à Belgrade, en rappelant que le combat pour le Kosovo sera un combat diplomatique et non armé. Pour l'occasion, environ 200000 Serbes, de toute la Serbie, s'étaient réunis dans la ville pour défiler entre le Parlement et l'Eglise Saint Sava à partir de 17 h00 jusqu'à 21 h00. En marge des cortèges, des incidents commencent devant l'Ambassade des Etats-Unis, à partir de $18 \mathrm{~h} 30$. Cette fois-ci, près de cinq cent casseurs sont recensés, les « hooligans » ayant été rejoints par des groupuscules d'extrême droite plus organisés. Les dégâts dans la ville sont plus conséquents. L'Ambassade des Etats-Unis (photo 5), ainsi que celle de Croatie voisine sont incendiées. Les Ambassades du Canada, de Bosnie-Herzégovine, de Belgique et d'Allemagne sont endommagées. Les deux Mac 
Donald du centre-ville sont à nouveau incendiés. Plusieurs boutiques locales et étrangères sont visées par les attaques et mises à sac parmi lesquelles Nike, Reebok, Benetton et des agences de la Société Générale (photo 6) et de la Raffeisen Bank. Les affrontements avec la police ont duré jusque tard dans la nuit et ont fait plus de cent blessés de part et d'autre, tandis qu'un manifestant mourrait dans l'incendie de Ambassade des Etats-Unis.

Photo 5 - L'Ambassade des Etats-Unis incendiée le 23 février 2008

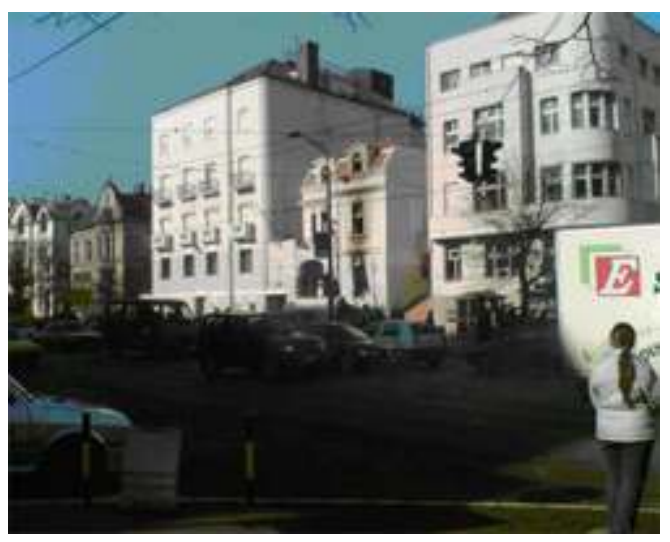

Photo 6 - Un distributeur de billet attaqué à la Société Générale

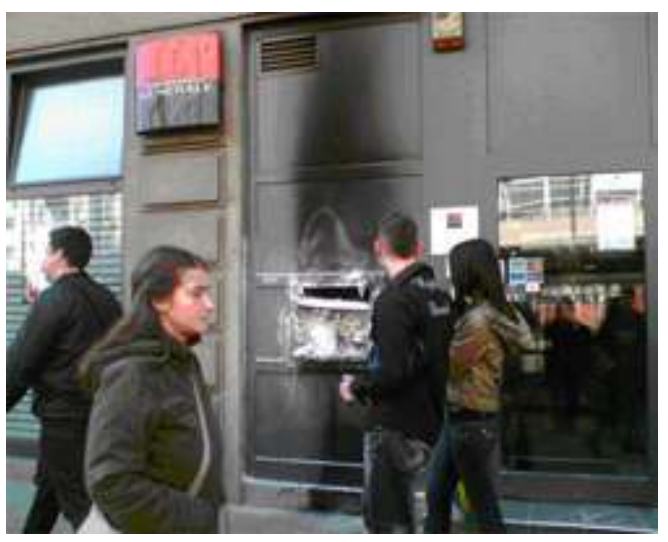

Précisons tout de suite que ces actes sont le fait d'infimes minorités et qu'ils sont aussitôt condamnés par les autorités serbes. Dès le lundi 18 février, des milliers de personnes ont manifesté pacifiquement à Belgrade (photo 7), entonnant des slogans comme "Kosmet je Srbija, Srbija je svet" (le Kosovo est la Serbie, la Serbie est le monde) ou "Kosovo je scrce Srbija" (le Kosovo est le coeur de la Serbie). Le jeudi 22 février, le cortège de manifestants fut, lui aussi, dans l'ensemble calme et non-violent. En tant que géographe, il est toutefois intéressant de suivre cette attention particulière aux lieux qui se développe aujourd'hui à Belgrade dans un contexte de crise. 
Photo 7 - Manifestation d'étudiants le lundi 18 février à Belgrade contre l'indépendance du Kosovo

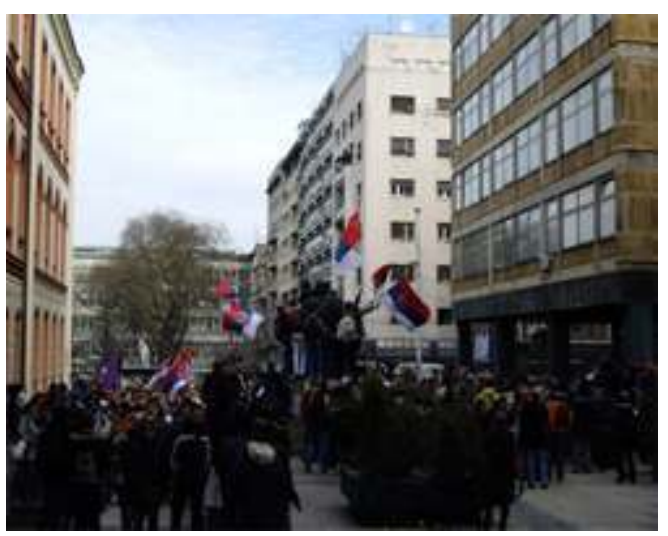

7 Ainsi, la perspective de cette destruction sélective, débutée quelques heures après l'annonce de la proclamation d'indépendance du Kosovo, avait inspiré, dans le courant de la semaine, une vague d'affichage nationaliste dans les boutiques du centre-ville. Dès le mercredi 21 février, anticipant les violences de la soirée du jeudi soir, certains commerçants locaux affichaient en vitrine de leur commerce le message Kosovo je Srbija [Le Kosovo est la Serbie], sous plusieurs formes allant du poster en couleur à une simple feuille de papier imprimée (photos 8 et 9). Le lendemain des manifestations, les commerces marqués étaient intacts, préservés des évènements, bien que les produits vendus par ces boutiques ne soient souvent pas d'origine locale. En réaction aux assauts sélectifs des "hooligans » contre des lieux identifiés comme le signe d'une ingérence étrangère indésirable, le marquage nationaliste permet au contraire de s'identifier à la cause serbe et de se protéger de ces casseurs extrémistes. Cette vague de destruction sélective a donc engendré, comme en écho, un marquage idéologique du paysage urbain belgradois. Les commerces qui ne sont pas directement assimilables à une firme étrangère, et qui pourraient être donc en situation d' "entre-deux ", de neutralité, ont bien souvent opté pour l'affichage nationaliste en vitrine, qui offrait une protection à moindre frais.

Photos 8 et 9 - Commerces locaux ayant affiché leur soutien à la manifestation du 22 février (cliché pris le 23 février 2008)

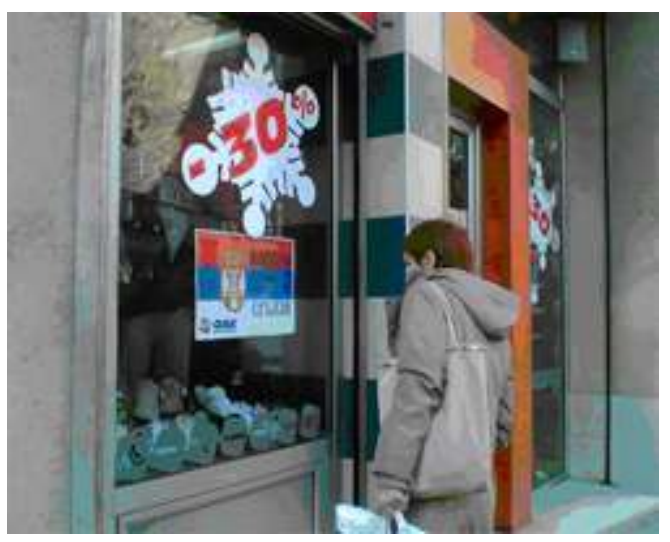




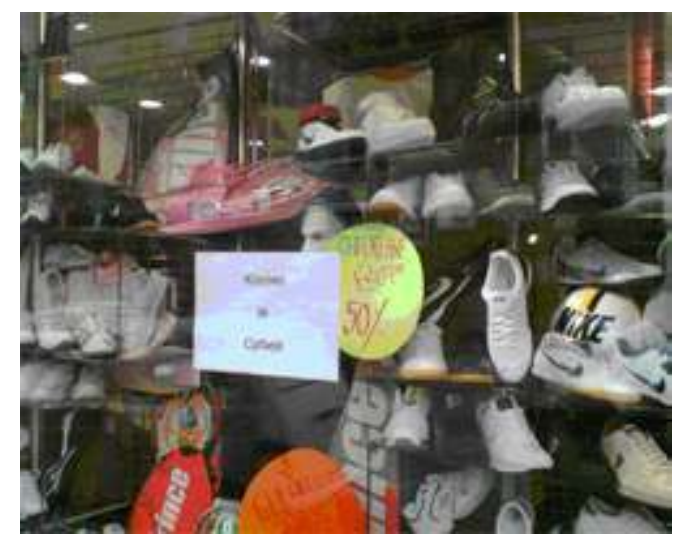

8 Parallèlement, en réponse à ces attaques sélectives, nous voyons s'instaurer une nouvelle géographie de la présence et de la visibilité policière dans la ville. Bien entendu, les policiers sont présents devant les ambassades particulièrement exposées (Etats-Unis, Turquie, Slovénie, France, Grande Bretagne, Allemagne, et plus généralement, de tous les pays qui ont déclaré aller vers une reconnaissance rapide de l'indépendance du Kosovo). Les forces de l'ordre contrôlent également de façon plus ou moins visible les principales routes d'accès aux mosquées et aux sites musulmans. Enfin, de façon plus inattendue mais compréhensible compte tenu de ce que nous venons d'expliquer, nous voyons aujourd'hui des policiers regroupés sur des sites plus inhabituels :

- autour de certains sites commerciaux étrangers les plus en vue, comme les centres commerciaux Mercator

- autour des restaurants Mac Donald's. Lundi 18 février, le Mac Donald de Nis apparaissait ainsi, dans les journaux télévisés du soir, entouré d'un cordon de police compact, le protégeant des attaques potentielles lors du passage du cortège de manifestants.

9 Ces quelques remarques mériteraient bien entendu une étude plus approfondie. Mais elles interpellent les géographes, sur la polysémie symbolique des lieux en fonction des contextes politique, social et économique. A vivre les évènements sur place à Belgrade, je pense qu'il y aurait là une thématique à creuser en géographie politique concernant le revirement brutal de la géographie symbolique et de l'espace vécu d'une ville en contexte de crise $^{3}$. Des sites symbolisant la coopération ou la porte (plus ou moins) ouverte vers l'étranger en temps normaux, comme peuvent l'être les ambassades, prennent une signification exactement inverse d'ingérence étrangère. Les lieux porteurs d'un message de coexistence multiculturelle, comme le sont les mosquées dans un environnement majoritairement orthodoxe, deviennent du jour au lendemain menacés, le message étant ici plus pernicieux puisqu'il assimile l'indépendance du Kosovo à une affaire non plus nationale mais religieuse. On retrouve là l'idée trompeuse de l'Islam comme une menace. Enfin, les sites qui ont pu signifier le développement, comme l'ont été les premiers centres commerciaux Mercator (le choix des consommateurs était enfin possible en Serbie) ou les premiers Mac Donald's, deviennent une immixtion économique illégitime de pays étrangers. 


\section{NOTES}

1. Politika est l'équivalent de Le Monde en terme de diffusion et de lectorat. Sa ligne éditoriale adopte des positions que l'on peut qualifier de nationalistes modérées.

2. Bien que le mot soit galvaudé en français, nous utilisons ici le terme « hooligan ", traduction directe du terme utilisé par la presse serbe, pour désigner ces jeunes casseurs, issus des groupes de supporteurs des équipes sportives de la région, souvent violents et ultranationalistes. De fait, les incidents ne sont pas rares lors des rencontres sportives. Pour une analyse plus précise du phénomène « hooligan » en ex-Yougoslavie, cf. JeanArnault Dérens, «L'éclatement de la Yougoslavie a commencé dans les stades », dans Sport et politique en Méditerranée, Chiclet, C.\& K. Gjeloshaj (dir.), l'Harmattan, 2005.

3. Cela à d'ailleurs déjà fait l'objet d'études assez précises et intéressantes concernant la ville de Sarajevo (voir l'article de Paul-David Régnier sur "Sarajevo, les géographies d'un siège" dans la revue Cités, n³2, "Sarajevo, l'Islam d'Europe").

\section{RÉSUMÉS}

La proclamation d'indépendance du Kosovo du 17 février entraînait immédiatement la réaction violente de groupe de jeunes «hooligans " à Belgrade. Leurs destructions sélectives des lieux révèlent un message politique clair contre les pays ayant prévu de reconnaître cette sécession. Ce contexte de crise produisait un revirement brutal de la géographie symbolique et de l'espace vécu dans la ville.

The Kosovo's independency proclaimed on the 17th February 2008 was immediately followed by violent reactions of young "hooligans" in Belgrade. Their selective destructions of places revealed a clear message against countries which planed to recognize this secession. This crisis context produced a brutal shift of the symbolical geography and of the daily experience of places in the town.

\section{INDEX}

Mots-clés : géopolitique, Serbie, crise politique, destruction urbaine, géographie symbolique Keywords : geopolitics, Belgrade, Serbia, political crisis, urban destruction, symbolical geography 


\section{AUTEUR}

\section{AMAËL CATTARUZZA}

(Monsieur) Amaël Cattaruzza est docteur en géographie, auteur d'une thèse intitulée "Le Monténégro entre Union et Indépendance. Essai sur une géographie du nationalisme" (2005 - Paris IV). Il est co-directeur de l'ouvrage "L'ex-Yougoslavie dix ans après Dayton" paru en 2005 à l'Harmattan. Il réside temporairement à Belgrade où il coordonne et anime un séminaire de recherches en sciences sociales à vocation régionale. 